\title{
La critique génétique et l'herméneutique ricœurienne
}

\section{Roberta Picardi}

\section{(2) OpenEdition}

1 Journals

\section{Édition électronique}

URL : https://journals.openedition.org/genesis/1793

DOI : 10.4000/genesis. 1793

ISSN : 2268-1590

\section{Éditeur :}

Presses universitaires de Paris Sorbonne (PUPS), Société internationale de génétique artistique littéraire et scientifique (SIGALES)

\section{Édition imprimée}

Date de publication : 9 mai 2017

Pagination : 197-202

ISBN : 979-1023-105636

ISSN : 1167-5101

\section{Référence électronique}

Roberta Picardi, «La critique génétique et l'herméneutique ricœurienne », Genesis [En ligne], 44 | 2017, mis en ligne le 22 mai 2018, consulté le 16 mars 2023. URL : http://journals.openedition.org/genesis/ 1793 ; DOI : https://doi.org/10.4000/genesis.1793 


\title{
La critique génétique et l'herméneutique ricœurienne
}

\author{
Roberta Picardi
}

L e rapport entre la génétique textuelle et l'œuvre de Ricœur peut être envisagé dans une double perspective. D'une part, il s'agit de considérer la relation entre la critique génétique et l'herméneutique ricœurienne du texte, en relevant les convergences et les divergences entre elles; d'autre part, il faut cerner les spécificités que l'œuvre de Ricœur présente du point de vue génétique.

\section{Intersections}

Le terrain de rencontre entre la critique génétique et l'herméneutique de Ricœur est constitué par la centralité qu'elles attribuent au processus de l'écriture : ce n'est pas un hasard si c'est avec une conférence intitulée «Regards sur l'écriture » que Ricœur intervient au colloque de critique génétique «La naissance du texte», organisé par Louis Hay en 19871 .

L'écriture constitue pour Ricœur «la toute première production », au-delà de laquelle il n'est pas possible de remonter, faute de retomber dans le psychologisme qu'il reproche - à tort ou à raison - à l'herméneutique romantique. C'est à partir de cette prémisse qu'il affirme :

[...] ce qui me paraît faire la force de la critique génétique, c'est qu'elle s'arrête aux premières traces matérielles de la création. Elle ne nous met jamais en face d'un psychisme en train de créer mais d'états successifs de la production. L'endeçà relève d'autre chose que de la critique; en ce sens, la critique génétique ne franchit pas la barrière qui la sépare d'une critique psychologisante ${ }^{2}$.

Plus précisément, le premier point de convergence entre la critique génétique et l'herméneutique ricœurienne peut être cerné dans l'identification entre écriture et création : «l'acte d'écrire » est pour Ricœur autre chose qu'un phénomène secondaire de fixation appliqué à un discours qui aurait pu être dit oralement. Il y a vraiment écriture lorsque le discours produit n'a jamais été prononcé oralement et surtout n'aurait pas pu l'être. [...] un nouvel instrument de discours et de pensée naît avec l'écriture ${ }^{3}$.

Cette identification entre écriture et création relève chez Ricœur du fait qu'à ses yeux l'écriture ne se réduit pas à la fonction extérieure de fixation d'un discours oral, visant à le mettre à l'abri de la destruction : l'écriture exerce plutôt pour Ricœur la fonction fondamentale d'autonomiser l'œuvre à la fois par rapport à l'intentionnalité finie de son auteur et par rapport au contexte sociologique et économique dans lequel l'œuvre surgit. C'est seulement ce décrochage de l'œuvre par rapport au monde de l'auteur qui lui permet de projeter un nouveau monde : à savoir, le monde de nos possibilités les plus propres ou le monde du texte, qui est une réalité autre par rapport à la réalité visée par le discours oral et par le dialogue.

C'est ce double décrochage, par rapport à l'auteur, d'une part, par rapport à la réalité visible, d'autre part, qui marque l'espace de création engendré par l'écriture ${ }^{4}$.

De façon significative, dans son article inédit «Génétique et théorie littéraire» - mis en ligne sur le site de l'ITEM en 2007 - Louis Hay cite avec une évidente approbation ces passages de Ricœur concernant le rapport entre écriture et création, qu'il inscrit dans le sillage de Valéry, en affirmant :

1.P. Ricœur, «Regards sur l'écriture», dans L. Hay (dir.), La Naissance du texte, Paris, José Corti, 1989, p. 213-220.

2.Ibid., p. 220.

3.Ibid., p. 213-214.

4.Ibid., p. 214. 
À la suite de Valéry, Paul Ricœur écrit : «du seul fait que le discours est écrit, il est porteur d'une histoire qui n'est plus celle de son auteur [...] cette disjonction entre dire et signifier constitue déjà un phénomène de production, une création $5[\ldots]$ ».

Un point d'intersection ultérieur entre critique génétique et herméneutique ricœurienne peut être cerné également dans l'idée d'un lien constitutif entre écriture et distance critique. Ricœur considère notamment le processus d'objectivation du discours dans l'écriture comme une condition indispensable afin de sauvegarder le moment de la critique et de la distanciation, qu'il voit par contre compromis dans l'herméneutique gadamérienne, qui érige le dialogue en paradigme de l'expérience herméneutique ${ }^{6}$. De même, Louis Hay insiste sur la distanciation à l'égard de son propre objet - rendu possible par l'écriture - comme une condition indispensable de la «critique génétique», en tant que «critique». Tout en reconnaissant que la critique génétique partage avec l'herméneutique de Dilthey et avec son Einfühlungstheorie «l'empathie» issue «d'une longue immergence dans le travail d'un auteur », Hay souligne surtout - en accord avec Ricœur - la différence entre la critique génétique et la théorie diltheyenne de la sympathie identifiante : la critique génétique n'est pas une théorie de l'identification - visant à l'oubli de soi dans la conscience de l'écrivain - mais plutôt une observation des traces objectivées d'un travail de production, dont on peut rendre compte seulement si le chercheur se trouve à distance de son objet, car cette distance est la condition de toute relation critique 7 .

\section{Divergences}

Les intersections entre l'herméneutique ricœurienne et la critique génétique du texte que l'on vient de souligner ne doivent pas conduire à négliger les divergences importantes, qui relèvent de la différence des notions de texte, sur lesquelles Ricœur et la critique génétique s'appuient. La notion de texte n'a en effet ni la même signification ni la même ampleur dans l'herméneutique de Ricœur et dans la critique génétique. Si cette dernière établit un lien constitutif entre texte et publication imprimée, pour Ricœur la notion de texte est définie avant tout par l'écriture, et même l'écriture ne l'épuise pas. La notion de texte est plutôt définie par l'intersection de cinq critères : 1) l'effectuation du langage comme discours, dont l'unité-base est la «phrase»; 2) l'effectuation du discours comme œuvre structurée, au sens de totalité finie et close, dont la composition est soumise à une forme de codification de genre, qui interagit avec la configuration unique du style individuel; 3) l'effectuation de l'œuvre comme écriture; 4) la projection du monde du texte; 5) l'œuvre de discours comme médiation de la compréhension de soi ${ }^{8}$.

Cette extension en ampleur de la notion de texte rend difficile - pour Ricœur - la reconnaissance d'un espace propre à la notion d'avant-texte, qui semble en quelque sorte engloutie par la notion de texte, une fois que celle-ci est définie par l'écriture plutôt que par la publication imprimée. C'est donc de façon non fortuite que Ricœur aborde la notion d'avant-texte en la reliant à celle d'intertextualité 9 , bien que conçue dans un sens très élargi :

Il me semble que celle-ci [la critique génétique] crée un cas remarquable d'intertextualité, en ce sens que la découverte des versions antérieures au texte définitif équivaut à la production de textes sous le texte, d'avant-textes, comme on a dit, lesquels sont des textes que personne n'a jamais lu bien souvent, surtout s'ils n'ont pas été destinés à la publication et appartiennent au secret de l'auteur 10 .

Dès lors, pour Ricœur, l'avant-texte est «un texte sous le texte », qui représente un cas - bien que très particulier - d'intertextualité. Cette connexion entre avant-texte et intertexte est rendue possible par le fait que Ricœur établit

5.L. Hay, «Génétique et théorie littéraire», ITEM [en ligne], mis en ligne le 29 janvier 2007 ; disponible sur <www.item.ens.fr/index. php?id=27136>.

6.P. Ricœur, «La fonction herméneutique de la distanciation», dans Du texte à l'action. Essais d'herméneutique, II, Paris, Éditions du Seuil, 1986, p. 101-117.

7. L. Hay, «Génétique et théorie littéraire», art. cité. Sur l'immunité de la critique génétique à l'égard de la «tentation du psychologisme», voir aussi L. Hay, «Critiques du manuscrit», dans La Naissance du texte, op. cit., p. 9-20 (en particulier p. 11).

8. P. Ricœur, «La fonction herméneutique de la distanciation», art. cité, p. 102.

9. Ricœur insiste sur le caractère instable de la notion d'intertextualité, afin d'éviter tout risque d'absolutisation de l'intertexte : l'intertextualité est toujours à penser en rapport avec son opposé, à savoir dans la relation singulière que le texte entretient avec la réalité dont il parle et qu'il découvre.

10. P. Ricœur, «Regards sur l'écriture», art. cité, p. 216. 
une équivalence entre la constitution de l'avant-texte et la production d'un nouveau texte. Cette équivalence contestable du point de vue des généticiens et contestée par Hay - répond toutefois de façon cohérente à l'exigence de replacer l'avant-texte dans la dialectique entre écriture et lecture, qu'il considère comme le noyau du processus herméneutique.

En produisant des textes nouveaux, la critique génétique produit en même temps de nouveaux lecteurs, en quelque sorte des lecteurs de palimpsestes, lesquels, comme ces sinopies qu'on découvre après la dépose d'une fresque, sont des objets nouveaux que personne n'avait vu auparavant et qui se créent un public étrange, le public critique ${ }^{11}$.

Cette exigence de replacer l'avant-texte dans la dialectique entre lecture et écriture s'appuie notamment sur la conviction - que Ricœur partage avec Gadamer - selon laquelle l'achèvement du parcours de sens, dont le texte n'est que le point de départ, advient seulement dans la lecture, au cours d'une dialectique entre la structure durable de l'œuvre et sa déstructuration - restructuration par le lecteur.

Tout cela permet de préciser davantage le rapport entre herméneutique ricœurienne et critique génétique. D'une part, Ricœur reconnaît - avec la critique génétique - l'importance de la déstabilisation du texte; d'autre part, il place le lieu et le moment de déstabilisation non pas dans l'avant-texte, mais plutôt dans le moment de la lecture. L'avant-texte, par contre, n'est pour Ricœur qu'un moment dans la téléologie du texte ${ }^{12}$, qui sert non pas à déstabiliser la version finale de l'œuvre, mais plutôt à restituer la signification du texte de façon non naïve, plus élaborée et donc plus savante.

[...] ces avant-textes, qui encore une fois sont des textes nouveaux, entrent en compétition avec l'auctoritas du dernier texte $[. .$.$] . Je ne pense pas que ce phénomène constitue le$ dernier mot sur la question. [...] la reconstitution des états préalables d'un texte peut contribuer à restituer au dernier texte une consistance nouvelle dans la mesure où ce dernier texte apparaît comme le résultat d'une histoire auquel il confère une sorte de téléologie; c'est bien vers le dernier texte que tous les avant-textes empilés pointent 13 .

Plutôt, la déstabilisation du texte est pour Ricœur un processus réglé qui a lieu au cours de la dialectique entre écriture et lecture, à laquelle correspond - au plan de la critique - une dialectique entre étude de genèse et théorie de la réception.

\section{L'œuvre de Ricœur à l'épreuve de la critique génétique}

Après avoir considéré la critique génétique à l'épreuve de l'herméneutique ricœurienne, il s'agit, en retour, de considérer l'œuvre de Ricœur à l'épreuve de la critique génétique, en amorçant quelques réflexions sur l'écriture philosophique de Ricœur.

Le premier fait qui s'impose à l'attention du généticien et sur lequel Ricœur lui-même a attiré l'attention - est le lien étroit entre le travail de rédaction des ouvrages et l'activité d'exposition orale exercée en tant que professeur, sollicité pour présenter les résultats partiels de ses recherches au cours de rencontres et d'événements honorifiques de différentes natures (colloques, séminaires, etc.). On songe par exemple à Soi-même comme un autre, qui est issu de la révision et de l'amplification des «Gifford Lectures » - professées par Ricœur en 1986; cette révision et cette amplification ont pris forme à leur tour au cours d'autres événements publics, que Ricœur ne manque pas de rappeler dans la page de «remerciements » qui ouvre le livre ${ }^{14}$. Cet entremêlement entre la rédaction des ouvrages et les conférences destinées au grand public - mais aussi à des spécialistes internationaux - doit être pris en considération pour comprendre les choix rhétoriques et langagiers qui caractérisent le style philosophique de Ricœur, dont on loue souvent la clarté et la plasticité.

En même temps, il ne faut pas sous-évaluer l'importance fondamentale que revêtent aussi, d'un point de vue génétique, les cours que Ricœur donne à l'université, en France et aux États-Unis, en tant que professeur de philosophie. L'enseignement joue en effet un rôle essentiel dans la constitution de l'écriture philosophique de Ricœur : les moments où Ricœur se trouve confronté à l'histoire de la philosophie sur un plan purement didactique - «en quelque

11. Ibid., p. 216-217.

12. Sur la question complexe et controverse du rapport entre critique génétique et «téléologie», on renvoie à A. Grésillon, Éléments de critique génétique. Lire les manuscrits modernes, Paris, CNRS Éditions, deuxième édition, 2016, p. 164-168.

13. P. Ricœur, «Regards sur l'écriture», art. cité, p. 217. Voir aussi p. 218-219.

14. P. Ricœur, Soi-même comme un autre, Paris, Éditions du Seuil, 1990, p. 9. 
sorte professionnellement, sans être en état d'entrevoir en quoi elle participe » à sa propre recherche 15 - sont très rares ; par contre, dans la plupart des cas il oriente sa pratique d'enseignement selon ses propres questionnements, de sorte que c'est souvent dans les notes pour la préparation des cours que l'on peut trouver les noyaux génétiques de ses ouvrages. Ce lien entre enseignement et conception d'un ouvrage ou textualisation ressort par exemple de manière très évidente dans le cas du cours sur «Le concept philosophique de volonté ». De façon non fortuite, le texte manuscrit de la première leçon de ce cours a été rangé par Ricœur lui-même dans le dossier intitulé «Plans pour une nouvelle philosophie

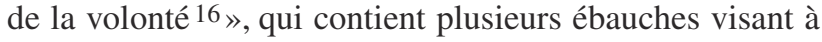
renouer et à renouveler le projet d'une philosophie de la volonté, qui avait inspiré ses premiers ouvrages. Bien que cette nouvelle «philosophie de la volonté»n'ait jamais été réalisée - de sorte que ces plans et les notes du cours sur le concept philosophique de volonté peuvent être considérés comme un «faux départ»-, on y trouve néanmoins les traces de la première genèse de plusieurs travaux successifs de Ricœur, de La Métaphore vive à la petite éthique de Soi-même comme un autre (voir fig. 1).

Cependant, pour comprendre la spécificité de l'écriture philosophique de Ricœur, on ne peut pas s'en tenir au simple constat du lien entre travail de rédaction et présentation orale : lien qu'on peut observer chez plusieurs auteurs. Il s'agit plutôt d'attirer l'attention sur un autre aspect, qui peut être identifié comme la véritable marque de fabrique de l'écriture philosophique de Ricœur : à savoir, l'ancrage de la rédaction et de la textualisation dans un travail extraordinaire de documentation prérédactionnelle. Les traces de ce travail de documentation se trouvent en amont et en aval de l'œuvre de Ricœur : en amont, dans les archives, où une masse considérable de documents conservés est représentée par les notes de lecture; en aval, dans le style typique de l'écriture philosophique de Ricœur, laquelle - surtout après son tournant herméneutique - est caractérisée par la tendance méthodique à passer en revue les grands penseurs du sujet traité, avant d'avancer ses propres hypothèses. Cette façon de travailler et ce style d'écriture philosophique reflètent un des traits distinctifs de l'herméneutique ricœurienne, à savoir ce que Ricœur lui-même a exprimé de façon très efficace en utilisant la célèbre métaphore de la « voie longue» ou de la «voie patiente», qu'il oppose notamment à la «voie courte» de l'herméneutique radicale et ontologisante de Heidegger et Gadamer. Le noyau de cette métaphore, que Ricœur utilise en plusieurs occasions, réside dans l'idée, inspirée par Jean Nabert, selon laquelle nous ne pouvons pas parvenir à la conscience de nous-même - et nous approprier le désir d'être qui nous constitue - si ce n'est à travers la voie longue de la médiation des signes et des symboles dans lesquels ce désir d'être s'est objectivé, selon un processus régi par des règles de «méthode» qu'il ne faut pas opposer à la «vérité». C'est cette idée qui nourrit un style philosophique - comme celui de Ricœur - qui se donne presque comme règle celle de traverser et de laisser traverser le lecteur à la fois les grandes traditions philosophiques et les sciences humaines, afin de développer et d'exposer sa propre position. La génétique textuelle se révèle comme une clé d'accès privilégiée dans cette «voie longue», car elle permet de saisir le véritable sens, les différentes étapes et le caractère dynamique de l'appropriation productive de la pensée d'autres auteurs, dont cette voie longue est nourrie.

15. P. Ricœur, «L'histoire de la philosophie et l'unité du vrai », dans Histoire et vérité, Paris, Éditions du Seuil, 1964, p. 64.

16. Archives Ricœur, Bibliothèque de l'Institut protestant de théologie, Classeur 12 : «Plans pour une nouvelle philosophie de la volonté», f. 31534-31600. 


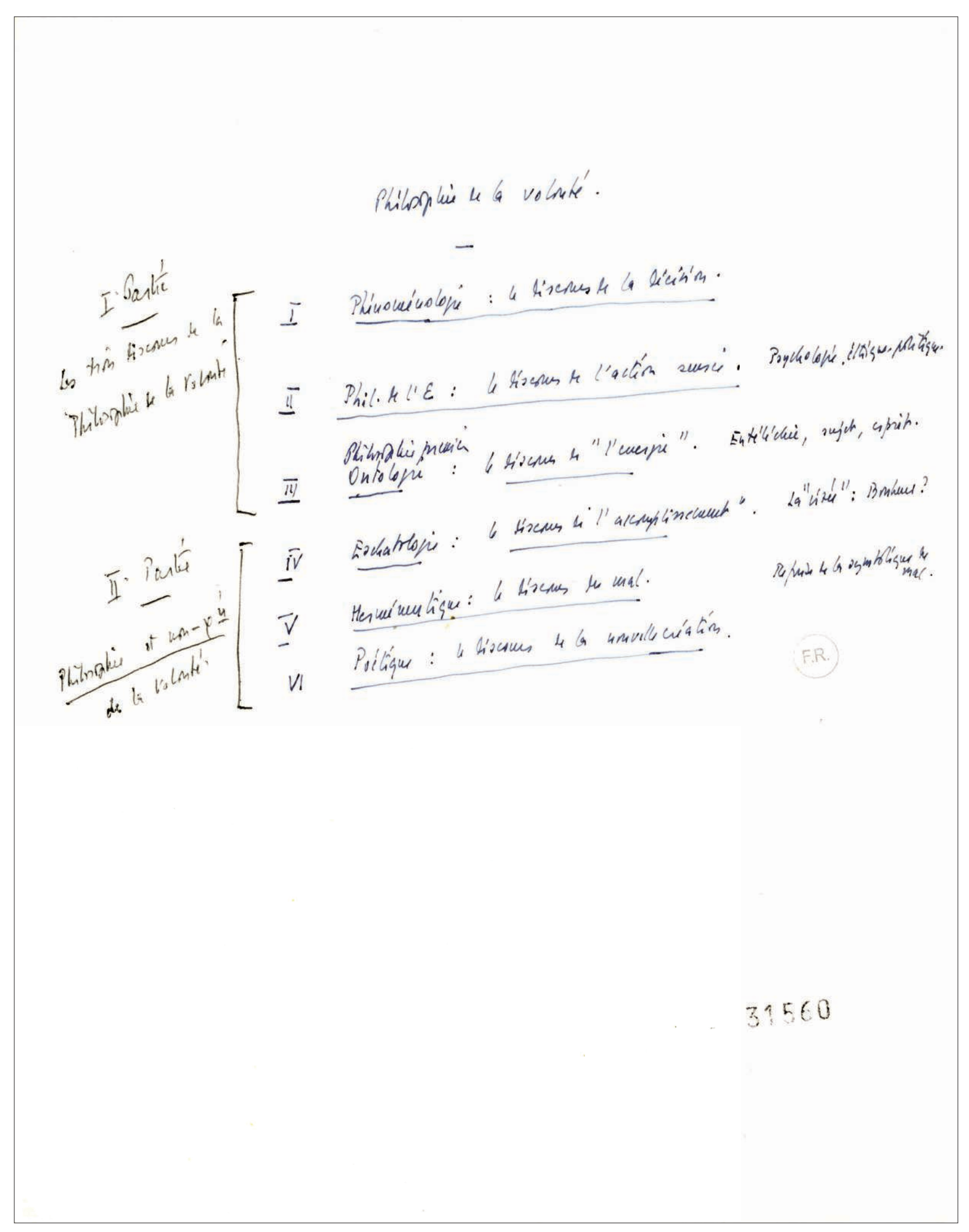

Fig. 1 : Paul Ricœur, feuillet extrait du dossier «Plans pour une nouvelle philosophie de la volonté » (Fonds Paul Ricœur, classeur 12, f. 31560) @ Comité éditorial du Fonds Ricœur 
Roberta PiCARDi est professeure à l'Université del Molise (Italie) et membre du conseil scientifique du fonds Ricœur. Ses travaux portent sur la philosophie classique allemande et sur son héritage dans la philosophie contemporaine. Elle a publié notamment une monographie sur la philosophie de l'histoire de Fichte (Il concetto e la storia, Bologna, il Mulino, 2009) et dirigé le numéro thématique de la Revue des sciences philosophiques et théologiques, «Paul Ricœur : de la volonté à l'action» (t. 99, 2015/4).

roberta_picardi@virgilio.it

\section{La critique génétique et l'herméneutique ricœurienne}

L'article envisage le rapport entre la génétique textuelle et l'œuvre de Ricœur dans une double perspective. D'une part, il s'agit de mettre à jour les convergences et les divergences entre la critique génétique et l'herméneutique ricœurienne du texte : si le principal point d'intersection réside dans l'identification entre écriture et création, les divergences relèvent par contre de la différence des notions de texte, sur lesquelles Ricœur et la critique génétique s'appuient; d'autre part, l'article se concentre sur les spécificités de l'œuvre de Ricœur du point de vue génétique : la marque distinctive de l'écriture philosophique de Ricœur est à cerner dans l'ancrage de la rédaction dans un travail extraordinaire de documentation prérédactionnelle; ce style d'écriture réfléchit à son tour la «voie longue» qui constitue notamment le trait distinctif de l'herméneutique ricœurienne par rapport à celle de Gadamer.

This article studies the connection between textual genetics and Ricœur's work in a double perspective. On the one hand we will show the convergences and divergences between critical genetics and Ricœur's hermeneutics of the text: If the main crossing point resides in the identification between writing and creation, divergences however are ascribable to the different notions of the text, on which Ricœur and critical genetics rely. On the other hand, this article focuses on the specificity of Ricœur's works from a genetic standpoint: The hallmark of Ricœur's philosophical writing is to be found in the draft's anchoring in an extraordinary pre-editorial documentation. This writing style reflects in turn the "long run" that constitutes in particular the distinctive features of Ricœur's hermeneutics compared to Gadamer's.

Der Artikel betrachtet die Beziehung zwischen Textgenese und Werk Ricœurs in einer zweifachen Perspektive. Einerseits geht es darum, die Konvergenzen und Divergenzen zwischen critique génétique und Ricœurscher Texthermeneutik aufzuzeigen: während der gemeinsame Punkt in den Begriffen von Schrift und Schaffen liegt, beruhen die Divergenzen auf unterschiedlichen Begriffen von „Text“", auf welche sich Ricœur und die critique génétique stützen. Ein zweiter Schwerpunkt des Artikels sind die besonderen Eigenschaften von Ricœurs Werk unter dem Gesichtspunkt der Textgenese: das unterscheidende Merkmal von Ricœurs philosophischem Schreiben ist seine Verankerung in einer ganz außerordentlichen prä-redaktionellen Dokumentationsarbeit; dieser Schreibstil reflektiert seinerseits den „langen Weg“, der bekanntlich den Hauptunterschied zwischen Gadamers und Ricœurs Hermeneutik ausmacht.

Este artículo estudia la relación entre la genética textual y la obra de Ricœur en una doble perspectiva. Por una parte se trata de establecer las convergencias y divergencias entre la crítica genética y la hermenéutica del texto de Ricœur: si el punto de intersección principal reside en la identificación entre escritura y creación, las divergencias dan cuenta, por el contrario, de la diferencia entre las nociones de texto en las que Ricœur y la crítica genética se basan; por la otra, el artículo se centra en las especificidades de la obra de Ricœur desde el punto de vista genético: la característica distintiva de la escritura filosófica de Ricœur hay que buscarla en el anclaje de la redacción en un trabajo extraordinario de documentación prerredaccional. Ese estilo de escritura refleja, a su vez, la "vía larga" que constituye, en particular, el rasgo distintivo de la hermenéutica de Ricœur en relación con la de Gadamer.

A genética textual e a obra de Ricœur são encaradas em perspectiva dupla. Por um lado, evidenciam-se convergências e divergências entre a crítica genética e a hermenêutica do texto de Ricœur: o principal ponto de intersecção encontra-se na identificação entre escrita e criação, mas as divergências acham-se na diferença entre os conceitos de texto; por outro lado, interessam as especificidades da obra de Ricoeur do ponto de vista genético: a marca distintiva da escrita filosófica de Ricœur encontra-se na dependência da sua redacção em relação ao extraordinário trabalho de documentação pre-redaccional, reflexo daquela "via longa" que distingue a hermenêutica de Ricœur da de Gadamer.

L'articolo affronta il rapporto tra la genetica testuale e l'opera di Ricœur in una duplice prospettiva. Da un lato, si tratta di mettere a fuoco le convergenze e le divergenze tra la critica genetica e l'ermeneutica ricoeuriana del testo: se il principale punto d'intersezione risiede nell'identificazione tra scrittura e creazione, le divergenze dipendono invece dalla differente nozione di testo, sulla quale si fondano l'ermeneutica ricoeuriana e la critica genetica. Dall'altro lato, vengono individuate le peculiarità dell'opera di Ricœur, dal punto di vista genetico: il tratto distintivo della scrittura filosofica ricoeuriana puo' essere identificato nell' ancoraggio della testualizzazione in un lavoro straordinario di documentazione pre-redazionale; questo stile di scrittura riflette a sua volta la "via lunga" che caratterizza l'ermeneutica di Ricœur rispetto a quella di Gadamer. 